\title{
INVESTMENT CASE \& EQUITY: A QUASI-EXPERIMENTAL ANALYSIS OF MATERNAL \& CHILD HEALTHCARE SERVICES IN PUNJAB
} Majid Ali $^{1^{*}}$, Naeem Ahmed Qureshi ${ }^{2}$, Ali Akbar Pirzado ${ }^{3}$, Iftakhar Ahmed ${ }^{4}$, Muhammad Rizwan Sabir ${ }^{5}$

${ }^{1 *}$ PMAS-ARID, Agriculture University, Rawalpindi, Punjab, Pakistan; ${ }^{2}$ Department of Statistics, Sindh Agriculture University, Tan do jam, Pakistan; ${ }^{3}$ Shaheed Mohtarma Benazir Bhutto Medical University, Larkana, Pakistan;

${ }^{4}$ Assistant professor in Statistics, Department of Economics Lasbela University of Agriculture Water and Marine Sciences (LUAWMS), Uthal, Balochistan, Pakistan; ${ }^{5} \mathrm{Ph} . \mathrm{D}$., Scholar, University of Lahore, Pakistan.

Email: ${ }^{1 *}$ majidali@uaar.edu.pk, ${ }^{2}$ qureshistat@gmail.com, ${ }^{3}$ pirzadoaa@gmail.com, ${ }^{4}$ iftkhanzada@gmail.com, 5ustrizig@gmail.com

Article History: Received on $26^{\text {th }}$ March 2021, Revised on $21^{\text {st }}$ April 2021, Published on $28^{\text {th }}$ April 2021

\begin{abstract}
Aim: This research aims to define equities determinants in maternal and child care in Punjab, a Pakistan province. The study focuses on the impact on Reproductive/ Maternal/ Newborn/ Child Adolescent Health (RMNCAH), introduced in 2016 through the Investment Case (IC) approach.
\end{abstract}

Methodology: A complex-sample-analysis modified the weight of the sample. Weighted disaggregated data were produced by cross-tableting with Confidence Interval (CI). A DiD analysis was performed based on a linear model of regression. Finally, the effect of the involvement was calculated by multivariate linear regression.

Principal Findings: The results show that changes had been measured in both the involvement and reference areas in involvement and no significant associations in the variables. Changes in involvement and contrast areas were similar. Also, there was no substantial improvement in aggregate outcomes from the multivariable regression analysis. In most developing countries, the IC approach is successful. Following the IC's introduction, some MNCH indicators such as ANC and the delivery of expert delivery of assistant birth showed improvements in involvement and contrast districts.

Applications of this study: The research will provide a proper guideline for the policymaker to design a need-based policy for equitable admission to child health care and maternal facilities

Novelty/Originality of this study: This study first analyzed the influence of investment- case in the RMNCAH program in Punjab.

Keywords: Child Healthcare, Maternal Analysis, RMNCAH, Weighted Disaggregated, Multivariable Regression Analysis.

\section{INTRODUCTION}

A global commitment to reducing maternal mortality has also been an essential share of the sustainability plan (Magni, 2017). Goal three purposes to facilitate the global mother mortality rate to less than 70 per 100,000 live births. (Geller et al., 2018). In developed countries, nearly all (99 percent) mother deaths occur (Thompson-Dudiak, 2021). The primary cause of death and disability in Pakistan remains pregnancy and birth complications (Nigussie et al., 2021). Pakistan is one of six countries with an annual mortality ratio of 297 to 100000 live births and more than 50 percent of all maternal deaths worldwide (Cabero-Roura \& Rushwan, 2014). Pakistan's formal maternal health policy reflects broadly endorsed strategies to promote safe childbirth, skilled birth attendance, and a timely emergency referral to a sound health system (Vogel et al., 2016). This policy was implemented with considerable efforts, first in the context of Safe Motherhood and later in MDGs, to improve service delivery (Patel et al., 2016).

Pakistan is unlikely to meet the fifth MDG objectives (Jin et al., 2018), which, despite these efforts, including reducing maternal mortality and access to universal reproductive health care (Dawson et al., 2014). One reason humans did not understand and address the factors limiting women who live on the economic and social margins of society's access to care (Khan et al., 2020). The most recent national data indicate that widespread maternal care socioeconomic inequalities have continued for all indicators (Novignon et al., 2019). Since this $92 \%$ of women report having antenatal care in the highest quintile wealth, $37 \%$ of women are in the lowest quintile rate (Ahinkorah et al., 2021). In the same way, $74 \%$ of women are present in the most significant higher-income households, particularly in contrast with $12 \%$ of women in the smallest quintile (El-Kak et al., 2020).

The current Pakistan NMN 2007 (Viswanath \& Kreuter, 2007) recognizes these inequalities and strives to ensure care for disadvantaged and vulnerable groups (Bhutta et al., 2013). To date, policies and practical measures continue to be "more equal," with a significant focus on enhancing district healthcare organizations through optimized technology and managerial ability and creating a new community skilled workers Structure (Heerdegen et al., 2020). However, efforts were made to increase demand for maternal health care using targeted, socially acceptable communications strategies in government (Fotso et al., 2015). A simple technical approach is not enough in terms of supply to address the discriminations to get the maternal healthcare services and in the maternal death levels reflected in this growing recognition (Okoli et al., 2020). 
At the local level, the RMNCAH Investment Case aims to establish a cohesive strategy for regional growth, equitable and sensitive bottlenecks, and local needs to develop health and nutrition for children and young people (Requejo et al., 2020). The Investment Case (IC) is a systematic approach supported by evidence that solves problems to encourage better health and budgeting of mothers, neonates, and children (Azad et al., 2021). It emphasizes the need to improve health MDGs 4 and 5 instantly by defining the health problems in the MNCH sector of a state (Liang et al., 2019). The IC research is based mainly on the 'Tanahashi' model, five different capabilities, and involvement predictors to achieve the desired service level (i.e., successful coverage) (Tibeihaho et al., 2021). The Tanahashi model was developed in 1978 to recognize gaps in the system's quality and effectiveness (McCollum et al., 2019). The difference is the percentage of the target group that is not effectively shielded.

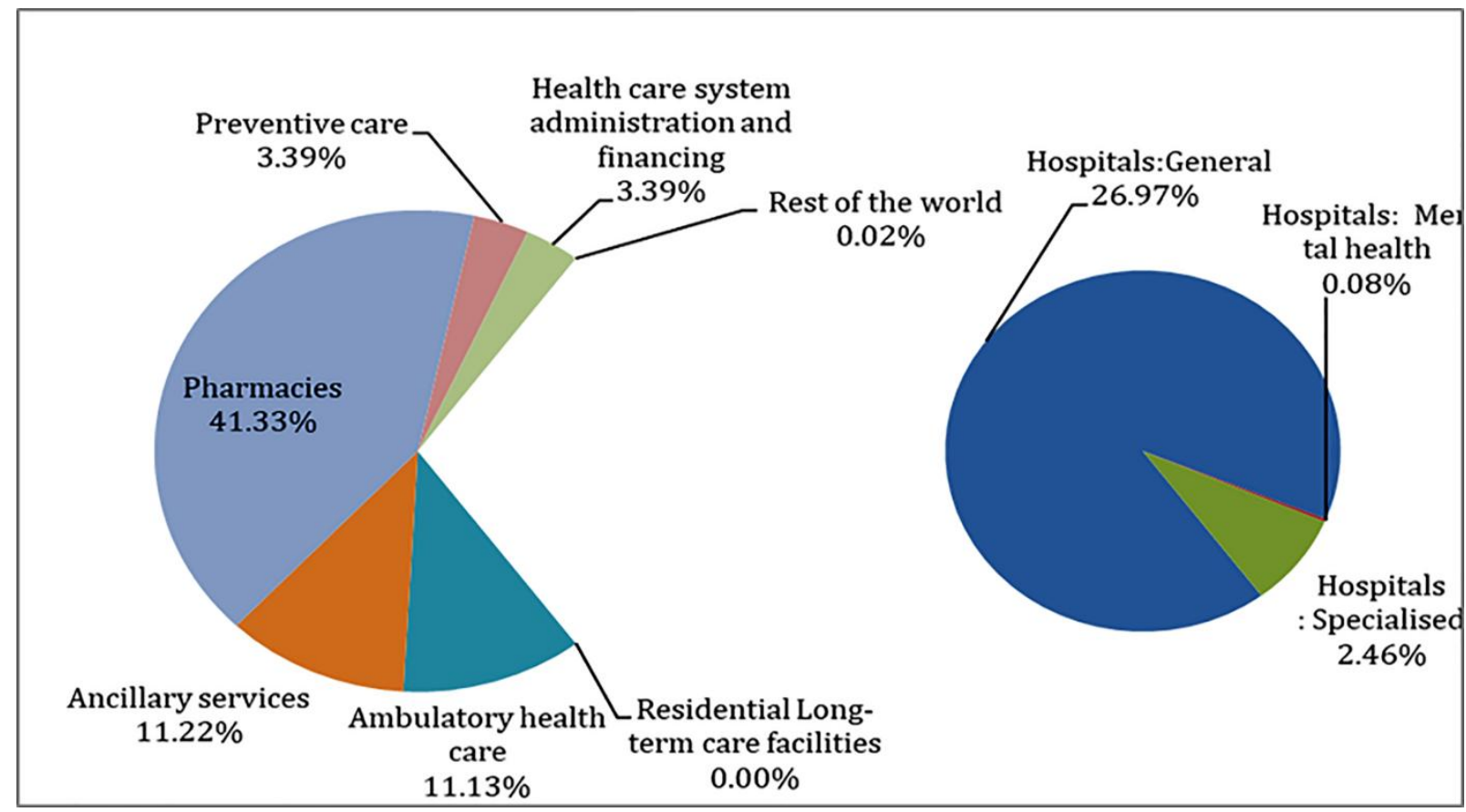

Figure 1: Healthcare Services in Pakistan

Source: Pakistan Development and Health Survey (PDHS)

There is officially no detailed understanding of long-term socioeconomic marginalization to restrict poor women have access to maternal health care services in Pakistan and because of their acknowledgment to caring for marginalized communities (Perry et al., 2017). To develop measures that address persistent inequalities, it is crucial to understand these structures and processes and identify the poor and socially excluded effectively (Mumtaz et al., 2014). We report a detailed qualitative study that starts dealing with these issues. Our findings provide a clearer picture of the factors which affect equal access in Punjab Pakistan (Gardezi, 2021) for maternal and children services. And highlight many vital aspects which need to be highlighted in developing and implementing plans and actions uncertainty inequalities in maternal health care to be addressed more effectively (Askew et al., 2019).

The Need and objective of the study

This study is important to identify the element to educate women about maternity and child health care, the RMNCAH, and to define the aspects that influence the decision making and implementation during maternity.

Keeping above in the mind the study focuses on the impact on Reproductive/ Maternal/ Newborn/ Child Adolescent Health (RMNCAH), introduced in 2016 through the Investment Case (IC) approach.

\section{METHODOLOGY}

\section{Punjab Population Study}

The province of Punjab, the most populous region of Pakistan and the second-largest province by area is divided into 36 districts and nine divisions, and its total population is 110,012,442 (Garcia et al., 2019). Population and environment of the study Punjab is divided geographically into three regions: central, southern, and northern (Mustafa et al., 2020). So, $63 \%$ of people are in rural areas, according to the 2017 census. The current study used the investment case strategy in 16 districts (Laing et al., 2020). The contrast was made in 20 communities with HDI (Panda et al., 2020). There were multiple areas for both involvement and contrast districts.

\section{Design of the Study}

The research used a quasi-experimental approach in the case of the investment case (IC) method to evaluate the effect 
on mother-and-child health services in Punjab (Tsolaki et al., 2020; Bärnighausen et al., 2017). The PDHS was used to measure the influence of the IC approach of the sixteen involvement and twenty contrast districts in Punjab (Thapa et al., 2020). Two surveys contain data, namely PDHS 2017 and 2019. Both survey methods were identical.

\section{Data Sources}

The Punjab population and healthcare analysis assess the involvement bundle established through the IC (Investment Case) method on child \& mother healthcare facilities in Punjab (Hao et al., 2020). The Multiple Indicator Cluster Survey (MICs) has a standardized analysis that accumulates statistics about the people, fitness, and diet of households. The MICS research is descriptive at the national level and uses a multi-stage analysis (Nisar \& Dibley, 2014). There are two polls, i.e., MICS 2017 and 2019. Both methods of the survey were similar. Due to the length of involvement, data on several prenatal cares, at least four prenatal care, and professional care is limited to the last three years (from 2016 to 2020), i.e., back to 2017 studies and back to 2019.

Table 1: No. of reproductive household and women age

\begin{tabular}{lll}
\hline Parameters & MICS 2017 & MICS 2019 \\
\hline Aggregate HH (household) & 11,835 & 12,564 \\
\hline Rate of Response (\%) & 98.3 & 99.6 \\
\hline 15-49 years of women ages & 13,774 & 14,101 \\
\hline Rata of Response $(\%)$ & 97.2 & 99.5 \\
\hline
\end{tabular}

\section{Conceptual Framework}

Figure 2 showed the variables analyzed in this study.

\begin{tabular}{|l|l|}
\hline Dependent Variables & \multicolumn{1}{|c|}{ Independent Variables } \\
\hline $\begin{array}{l}\text { Program Involvement } \\
\text { (IC Approach) }\end{array}$ & $\begin{array}{l}\text { • Any ANC } \\
\text { - Involvement area }\end{array}$ \\
\hline - Contrast area & $\begin{array}{l}\text { - Expert delivery of } \\
\text { assisting the birth }\end{array}$ \\
\hline
\end{tabular}

Controlled Variables

Socio-economic variables: Education of Women's, Ages of Women, Household Head; Occupation of Women's; Ethnicity, Education of Husband, Wealth tertile

Access to health services: Distance to health care facilities closest,

Contextual factors: Residence Place, Organic area;

Figure 2: Conceptual Framework

\section{Data Analysis}

Any differences between the involvement and the contrast group were measured by DiD analysis, based on time changes rather than as a result of the participation itself (Gardezi, 2021). DiD Analysis was calculated using the linear regression method (Hashmi et al., 2021). Other variables indicate higher gains relative to districts except for utilities or corporations, the richest and hilly areas. Age groups 15-24 and 25-35, schooling, lowest and middle tertiary wealth, and married are significant advancements in districts. Other factors have improved by a moderate to minimal amount as compared to communities. Only the service and company categories' variations were statistically relevant because of at least four ANC (Smith et al., 2021). Before implementing the multivariate framework, multilinearity was evaluated for every model. Multicollinearity was assessed based on the three cut-off value variance inflation factors (VIF). The 
variables p-value influence 0.05 (first removed variables with the highest p-value) were ruled out one by one (Thompson et al., 2017). The final model was taken into account to reflect the real effects of involvement. For each variable, the process was repeated (Mauricio, 2018). The PHRCEC endorsed the Ethics Report.

\section{FINDINGS}

\section{DiD for a minimum of four ANC}

Table 2 shows differential (DiD) outputs about the allocation of female exogenous factors by participating and contrast region, due to the minimum 4 ANC practitioners' involving period. Minimum four ANCs increased in involvement after the participation, the value 41.3-55.8 and the contrast area 42.8-65.7. The difference in the rate of increase of 4.8\% was statistically insignificant. The contrast area Far from all variables, both involvement and contrast districts have improved. District enhancements seem to be more prevalent for most of the variables than the participating districts.

Except for service or companies, the highest income quintiles, and hilly areas, other variables indicate a more significant gain than districts. Age groups 15-24 and 25-35, schooling, lowest and middle tertiary wealth, and married are substantial advancements in districts. Other factors have improved by a moderate to minimal amount as compared to districts. Only the service and company categories' variations were statistically relevant because there were at least four ANC.

Table 2: DiD analysis minimum four ANC

\begin{tabular}{|c|c|c|c|c|c|c|c|c|c|c|}
\hline \multirow[t]{2}{*}{ Minimum 4 ANC } & \multicolumn{2}{|c|}{ Involvement area } & \multicolumn{3}{|c|}{ Contrast area } & \multirow{2}{*}{ N Difference } & \multirow{2}{*}{ DiD } & \multirow[t]{2}{*}{ Beta } & \multirow[t]{2}{*}{$\begin{array}{l}\text { CI } \\
(95 \%) \\
\end{array}$} & \multirow[t]{2}{*}{$\begin{array}{l}\text { P- } \\
\text { value }\end{array}$} \\
\hline & $\begin{array}{c}\text { Before } \\
\mathrm{N}=600 \\
\text { weighted }\end{array}$ & $\begin{array}{c}\text { After } \\
\mathrm{N}=603 \\
\text { weighted }\end{array}$ & Diff & $\begin{array}{c}\text { Before } \\
\text { N=792 } \\
\text { weighted }\end{array}$ & $\begin{array}{l}\text { After } \\
\quad=740 \\
\text { weighted }\end{array}$ & & & & & \\
\hline \multirow[t]{2}{*}{ Minimum 4 ANC } & 41.3 & 55.8 & 14.5 & 42.8 & 65.7 & 22.9 & -4.8 & -0.006 & $-0.031-$ & 0.547 \\
\hline & & & & & & & & & 0.022 & \\
\hline \multicolumn{11}{|l|}{3 cat Age } \\
\hline \multirow[t]{2}{*}{15 to 24 years } & 44.63 & 54.69 & 7.3 & 55.31 & 61.75 & 12.7 & -1.3 & -0.007 & $-0.056-$ & 0.795 \\
\hline & & & & & & & & & 0.029 & \\
\hline \multirow[t]{2}{*}{25 to 34 years } & 37.04 & 52.12 & 15.4 & 37.26 & 55.67 & 19.8 & -6.4 & -0.014 & $-0.068-$ & 0.559 \\
\hline & & & & & & & & & 0.026 & \\
\hline \multirow[t]{2}{*}{35 to 49 years } & 18.06 & 35.77 & 16.5 & 24.55 & 56.46 & 27.2 & -11.5 & -0.071 & $-0.068-$ & 0.479 \\
\hline & & & & & & & & & 0.037 & \\
\hline \multicolumn{11}{|l|}{ Culture } \\
\hline \multirow[t]{2}{*}{ Pros } & 51.54 & 63.19 & 12.7 & 52.56 & 73.59 & 21.9 & -7.3 & -0.088 & $-0.047-$ & 0.346 \\
\hline & & & & & & & & & 0.020 & \\
\hline \multirow[t]{2}{*}{ Cons } & 35.20 & 47.85 & 11.5 & 36.40 & 55.22 & 17.6 & -3.0 & -0.009 & $-0.055-$ & 0.673 \\
\hline & & & & & & & & & 0.029 & \\
\hline
\end{tabular}

Education

\begin{tabular}{|c|c|c|c|c|c|c|c|c|c|}
\hline No Education & 31.20 & 42.55 & 12.5 & 18.63 & 45.77 & 26.2 & $-13.6-0.021$ & $-0.056-$ & 0.089 \\
\hline & & & & & & & \multicolumn{3}{|c|}{0.005} \\
\hline \multirow[t]{2}{*}{ Primary } & 53.33 & 44.80 & -9.4 & 45.70 & 55.70 & 5.0 & $-17.4-0.059$ & $-0.078-$ & 0.226 \\
\hline & & & & & & & \multicolumn{3}{|c|}{0.018} \\
\hline \multirow[t]{2}{*}{ Higher Education } & 72.86 & 73.33 & 2.4 & 65.65 & 71.05 & 5.6 & $-6.3-0.007$ & $-0.025-$ & 0.554 \\
\hline & & & & & & & \multicolumn{3}{|c|}{0.026} \\
\hline \multicolumn{10}{|l|}{ Profession } \\
\hline \multirow[t]{2}{*}{ Jobless } & 42.37 & 44.88 & 4.3 & 43.75 & 55.77 & 5.3 & $\cdot 1.1-0.004$ & $-0.066-$ & 0.831 \\
\hline & & & & & & & \multicolumn{3}{|c|}{0.051} \\
\hline \multirow[t]{2}{*}{ Agriculture work } & 38.42 & 56.26 & 16.8 & 35.57 & 65.10 & 22.5 & $\begin{array}{ll}-5.7 & -0.023\end{array}$ & $-0.065-$ & 0.3545 \\
\hline & & & & & & & \multicolumn{3}{|c|}{0.019} \\
\hline \multicolumn{2}{|c|}{ Service or business 35.10} & 85.78 & \multicolumn{2}{|c|}{44.1175 .25} & 71.26 & $\cdot 1.1$ & 46.10 .084 & $0.069-$ & 0.004 \\
\hline
\end{tabular}




\begin{tabular}{|c|c|c|c|c|c|c|c|c|c|}
\hline & & & & & & & \multicolumn{3}{|c|}{0.159} \\
\hline \multicolumn{10}{|c|}{ Wealth Tertlle } \\
\hline \multirow[t]{2}{*}{ Low } & 33.50 & 52.40 & 17.9 & 25.70 & 56.40 & 32.6 & $-15.9-0.030$ & $-0.076-$ & 0.119 \\
\hline & & & & & & & \multicolumn{3}{|c|}{0.007} \\
\hline Middle & 35.70 & 51.80 & 15.4 & 43.01 & 52.02 & 17.6 & $\begin{array}{ll}-3.3 & -0.009\end{array}$ & $\begin{array}{l}-0.051- \\
0.023\end{array}$ & 0.694 \\
\hline \multirow[t]{2}{*}{ High } & 52.60 & 55.40 & 3.9 & 75.20 & 71.80 & $\cdot 3.6$ & $\begin{array}{ll}6.4 & 0.041 \\
\end{array}$ & $-0.048-$ & 0.571 \\
\hline & & & & & & & \multicolumn{3}{|c|}{0.061} \\
\hline
\end{tabular}

Organic Region

\begin{tabular}{|c|c|c|c|c|c|c|c|c|c|c|}
\hline Central & 52.55 & 74.33 & 22.1 & 43.17 & 62.20 & 18.1 & 3.0 & 0.003 & $-0.042-$ & 0.927 \\
\hline & & & & & & & & & 0.040 & \\
\hline \multirow[t]{2}{*}{ Southern } & 36.66 & 52.23 & 12.3 & 56.08 & 73.54 & 18.4 & -5.0 & -0.009 & $-0.066-$ & 0.737 \\
\hline & & & & & & & & & 0.039 & \\
\hline \multirow[t]{2}{*}{ Northern } & 34.80 & 45.79 & 13.3 & 32.69 & 54.42 & 16.8 & -2.6 & -0.008 & $-0.055-$ & 0.902 \\
\hline & & & & & & & & & 0.050 & \\
\hline \multicolumn{11}{|l|}{ Residence \{U/R) } \\
\hline \multirow[t]{2}{*}{ Urban } & 52.44 & 55.65 & 3.3 & 54.62 & 67.6 & 8.1 & -5.7 & -0.008 & $-0.033-$ & 0.544 \\
\hline & & & & & & & & & 0.028 & \\
\hline \multirow[t]{2}{*}{ Rural } & 33.7 & 33.57 & 14.9 & 35.44 & 56.66 & 25.9 & $\cdot 12.1$ & $1-0.042$ & $-0.043-$ & 0.689 \\
\hline & & & & & & & & & 0.019 & \\
\hline \multicolumn{11}{|c|}{ Education of Husband } \\
\hline \multirow[t]{2}{*}{ No Education } & 2112.64 & 37.08 & 13.5 & 14.61 & 39.64 & 23.8 & -7.4 & -0.027 & $-0.079-$ & 0.534 \\
\hline & & & & & & & & & 0.025 & \\
\hline \multirow[t]{2}{*}{ Primary } & 42.37 & 43.89 & 0.9 & 37.85 & 57.79 & 18.6 & -1.9 & -0.064 & $-0.085-$ & 0.115 \\
\hline & & & & & & & & & 0.007 & \\
\hline \multirow[t]{2}{*}{ Higher Education } & 54.07 & 63.78 & 6.8 & 54.89 & 67.74 & 12 & -4.1 & -0.007 & $-0.039-$ & 0.610 \\
\hline & & & & & & & & & 0.025 & \\
\hline \multicolumn{11}{|l|}{ Household Head } \\
\hline \multirow[t]{2}{*}{ Male } & 36.44 & 51.36 & 11.6 & 42.65 & 58.76 & 15.4 & -4.8 & -0.008 & $-0.047-$ & 0.757 \\
\hline & & & & & & & & & 0.026 & \\
\hline \multirow[t]{2}{*}{ Female } & 49.93 & 61 & 11.2 & 45.87 & 66.8 & 17.9 & -9.8 & -0.027 & $-0.067-$ & 0.386 \\
\hline & & & & & & & & & 0.022 & \\
\hline \multicolumn{11}{|l|}{$\begin{array}{l}\text { Health Facilities } \\
\text { distance }\end{array}$} \\
\hline \multirow[t]{2}{*}{ high Problem } & 34.66 & 54.89 & 16.2 & 32.42 & 57.21 & 22.1 & -5.3 & $-(0.021$ & )$-(0.045)$ & $-(0.494)$ \\
\hline & & & & & & & & & 0.017 & \\
\hline Very low Problem & 48.36 & 56.31 & 1.3 & 59.21 & 63.43 & 8.2 & -6.0 & -0.033 & $-0.056-$ & 0.546 \\
\hline
\end{tabular}

\section{Expert delivery of assisting birth DID Analysis}

Table 3 illustrates the difference in variations and the importance of delivery by assisting birth inside the independent variables. Expert delivery of helping birth delivery in the involvement area has increased from 30.1 to 51.0 and in the contrasts between 28.8 and 43.1 following the involvement period. The variation in the increasing rate in the area of the involvement was $7.1 \%$ higher. Far from all factors, both involvement and contrast districts have improved. The variables showing the most significant changes in expert delivery of assisting birth's intervening districts are age 25-34 (difference 8,0), 35-49 (difference 15,8), advantaged ethnicity (difference 15.1), secondary or higher education, service, or business. Expert delivery of assisting birth has the highest age ranging and region of Hilly in the ecological areas. More improvements in the delivery of expert delivery of helping birth in involvement areas have also been seen in other variables, such as education of husband, residence, household head, and Distance to health care facilities closest. With a p-Value of 0.002 , it was statistically significant only that difference between the hill region category and the ecological region. Other variables such as primary education were almost meaningful Education for husbands (p- 
Humanities \& Social Sciences Reviews elSSN: 2395-6518, Vol 9, No 2, 2021, pp 475-486

https://doi.org/10.18510/hssr.2021.9246

value: 0.061 ). There have been no moderate increases in the contrast group in only plain regions (difference 5.0), no training in husband education. The DiD analysis has no significant variables with expert delivery of assisting birth's deliveries.

Table 3: DiD Analysis of expert delivery of assisting the birth

\begin{tabular}{|c|c|c|c|c|c|c|c|c|c|c|}
\hline \multirow[t]{2}{*}{$\begin{array}{l}\text { Skilled birth } \\
\text { attended }\end{array}$} & \multicolumn{3}{|c|}{ Involvement area } & \multicolumn{3}{|c|}{ Contrast area } & \multirow{2}{*}{$\begin{array}{c}\text { Differen } \\
\text { ce of } \\
\text { Differen } \\
\text { ce } \\
\end{array}$} & \multirow{2}{*}{$\begin{array}{c}\text { Beta } \\
\text { Coeff } \\
\cdot\end{array}$} & \multirow[t]{2}{*}{$\begin{array}{c}95 \% \\
\text { CI }\end{array}$} & \multirow[t]{2}{*}{$\begin{array}{c}P \text { - } \\
\text { value }\end{array}$} \\
\hline & $\begin{array}{c}\text { Before } \\
\mathrm{N}=679 \\
\text { weighte } \\
\text { d }\end{array}$ & $\begin{array}{c}\text { After } \\
\mathbf{N}=603 \\
\text { weighte } \\
\text { d }\end{array}$ & $\begin{array}{c}\text { Differen } \\
\text { ce }\end{array}$ & $\begin{array}{c}\text { Before } \\
\mathrm{N}=848 \\
\text { weighte } \\
\mathrm{d}\end{array}$ & $\begin{array}{c}\text { After } \\
\mathrm{N}=740 \\
\text { weighte } \\
\mathrm{d}\end{array}$ & $\begin{array}{c}\text { Differen } \\
\text { ce }\end{array}$ & & & & \\
\hline $\begin{array}{l}\text { Skilled } \\
\text { attended } \\
\text { delivery }\end{array}$ & 30.1 & 51 & 20.9 & 28.8 & 43.1 & 14.3 & 6.6 & 0.013 & $\begin{array}{l}- \\
0.010 \\
- \\
\end{array}$ & $\begin{array}{l}0.32 \\
5\end{array}$ \\
\hline & & & & & & & & & 0.039 & \\
\hline \multicolumn{11}{|l|}{ Age (3 cat) } \\
\hline \multirow[t]{2}{*}{$15-24$ yrs } & 33.54 & 52.89 & 21.5 & 31.16 & 60.79 & 15.5 & 5.6 & 0.009 & $\begin{array}{l}- \\
0.024 \\
- \\
\end{array}$ & $\begin{array}{l}0.58 \\
6\end{array}$ \\
\hline & & & & & & & & & 0.043 & \\
\hline \multirow[t]{2}{*}{$25-34$ yrs } & 27.25 & 45.31 & 18.1 & 27.43 & 37.49 & 10.1 & 8.0 & 0.016 & $\begin{array}{l}- \\
0.015 \\
-\end{array}$ & $\begin{array}{l}0.30 \\
6\end{array}$ \\
\hline & & & & & & & & & 0.047 & \\
\hline \multirow[t]{2}{*}{-49 years } & 13.81 & 42.12 & 19.2 & 15.37 & 17.93 & 3.1 & 14.1 & 0.127 & -0.16 & $\begin{array}{l}0.23 \\
3 \\
\end{array}$ \\
\hline & & & & & & & & & 0.084 & \\
\hline \multicolumn{11}{|l|}{ Ethnicity } \\
\hline \multirow[t]{2}{*}{ Benefits } & 24.61 & 61.20 & 27.3 & 41.26 & 61.74 & 21.1 & 13.5 & 0.131 & $\begin{array}{l}- \\
0.121 \\
\end{array}$ & $\begin{array}{l}0.41 \\
1 \\
\end{array}$ \\
\hline & & & & & & & & & 0.067 & \\
\hline Dis-adv & 32.33 & 50.49 & 18.2 & 24.20 & 38.45 & 14.2 & 3.9 & 0.007 & $\begin{array}{l}- \\
0.025 \\
-\end{array}$ & $\begin{array}{l}0.63 \\
6\end{array}$ \\
\hline \multicolumn{11}{|l|}{$\begin{array}{l}\text { Level of } \\
\text { Education }\end{array}$} \\
\hline \multirow[t]{2}{*}{ Illiterate } & 31.42 & 41.16 & 13.4 & 12.41 & 41.21 & 17.2 & -13.4 & $\begin{array}{l}- \\
0.025 \\
\end{array}$ & $\begin{array}{l}- \\
0.024 \\
\end{array}$ & $\begin{array}{l}0.75 \\
0 \\
\end{array}$ \\
\hline & & & & & & & & & 0.026 & \\
\hline \multirow[t]{2}{*}{ Primary } & 36.24 & 61.14 & 13.8 & 21.50 & 34.10 & 12.5 & 4.1 & 0.006 & $\begin{array}{l}- \\
0.041 \\
- \\
\end{array}$ & $\begin{array}{l}0.78 \\
1\end{array}$ \\
\hline & & & & & & & & & 0.052 & \\
\hline \multirow[t]{2}{*}{$\begin{array}{l}\text { Secondar/ } \\
\text { higher }\end{array}$} & 51.04 & 56.20 & 13.5 & 51.24 & 51.64 & 4.1 & 8.0 & 0.006 & $\begin{array}{l}- \\
0.021\end{array}$ & $\begin{array}{l}0.31 \\
5 \\
\end{array}$ \\
\hline & & & & & & & & & 0.041 & \\
\hline \multicolumn{11}{|l|}{ Job status } \\
\hline \multirow[t]{2}{*}{ Unemployed } & 37.18 & 46.21 & 13.1 & 41.05 & 51.13 & 7.0 & 4.0 & 0.004 & $\begin{array}{l}- \\
0.024\end{array}$ & $\begin{array}{l}0.65 \\
0\end{array}$ \\
\hline & & & & & & & & & 0.031 & \\
\hline \multirow[t]{2}{*}{$\begin{array}{l}\text { Agriculture } \\
\text { work }\end{array}$} & 17.64 & 41.89 & 29.7 & 22.67 & 36.95 & 15.0 & 6.3 & 0.014 & $\begin{array}{l}- \\
0.013 \\
-\end{array}$ & $\begin{array}{l}0.30 \\
8\end{array}$ \\
\hline & & & & & & & & & 0.043 & \\
\hline
\end{tabular}




\begin{tabular}{|c|c|c|c|c|c|c|c|c|c|c|}
\hline $\begin{array}{ll}\begin{array}{l}\text { Service } \\
\text { business }\end{array} & \text { or }\end{array}$ & 45.36 & 81.96 & 36.6 & 65.43 & 65.05 & -0.4 & 37.0 & 0.074 & $\begin{array}{l}- \\
0.006 \\
-\end{array}$ & $\begin{array}{l}0.07 \\
1\end{array}$ \\
\hline & & & & & & & & & 0.015 & \\
\hline \multicolumn{11}{|l|}{ Wealth Turtle } \\
\hline Lowest & 14.10 & 47.40 & 33.3 & 12.10 & 31.10 & 19.0 & 14.3 & 0.029 & $\begin{array}{l}- \\
0.004 \\
-\end{array}$ & $\begin{array}{l}0.08 \\
7\end{array}$ \\
\hline & & & & & & & & & 0.062 & \\
\hline Middle & 30.70 & 48.30 & 17.6 & 27.10 & 47.90 & 20.8 & -3.2 & $\overline{0} .006$ & $\begin{array}{l}- \\
0.042 \\
- \\
\end{array}$ & $\begin{array}{l}0.72 \\
5\end{array}$ \\
\hline & & & & & & & & & 0.029 & \\
\hline Highest & 50.70 & 59.80 & 9.1 & 56.50 & 69.30 & 12.8 & -3.7 & $\overline{-} 0.007$ & $\begin{array}{l}- \\
0.055 \\
-\end{array}$ & $\begin{array}{l}0.75 \\
7\end{array}$ \\
\hline & & & & & & & & & 0.040 & \\
\hline \multicolumn{11}{|l|}{ Organic Area } \\
\hline Southern & 22.56 & 76.09 & 45.4 & 27.15 & 35.95 & 11.4 & 34.7 & 0.067 & $\begin{array}{l}0.026 \\
-\end{array}$ & $\begin{array}{l}0.00 \\
2 \\
\end{array}$ \\
\hline & & & & & & & & & 0.108 & \\
\hline Northern & 17.04 & 39.92 & 22.9 & 28.95 & 43.43 & 14.5 & 8.4 & 0.016 & $\begin{array}{l}- \\
0.045 \\
- \\
\end{array}$ & $\begin{array}{l}0.59 \\
1\end{array}$ \\
\hline & & & & & & & & & 0.079 & \\
\hline Central & 26.71 & 37.41 & 21.5 & 31.41 & 35.21 & 21.4 & -3.1 & $\overline{0}-001$ & $\overline{0}-003$ & $\begin{array}{l}0.65 \\
4\end{array}$ \\
\hline & & & & & & & & & 0.041 & \\
\hline \multicolumn{11}{|c|}{ Area of Residence } \\
\hline Urban & 34.15 & 56.23 & 22.2 & 44.78 & 54.6 & 11.7 & 8.5 & $\begin{array}{l}0 . \mathrm{E} 01 \\
9\end{array}$ & $\begin{array}{l}- \\
0.020 \\
-\end{array}$ & $\begin{array}{l}0.33 \\
6\end{array}$ \\
\hline & & & & & & & & & 0.059 & \\
\hline Rural & 26.51 & 44.8 & 18.3 & 15.43 & 32.47 & 17 & 1.3 & 0.002 & $\begin{array}{l}- \\
0.029 \\
-\end{array}$ & $\begin{array}{l}0.87 \\
6\end{array}$ \\
\hline & & & & & & & & & 0.034 & \\
\hline \multicolumn{11}{|c|}{ Education of Husband } \\
\hline $\begin{array}{l}\text { No } \\
\text { Education }\end{array}$ & 25.32 & 35.65 & 13.8 & 11.45 & 32.51 & 24.7 & -6.7 & 0.013 & $\begin{array}{l}- \\
0.056 \\
-\end{array}$ & $\begin{array}{l}0.55 \\
3\end{array}$ \\
\hline & & & & & & & & & 0.030 & \\
\hline Primary & 40.23 & 29.16 & 28.2 & 32.60 & 41.3 & 17.4 & 19.3 & 0.000 & $\begin{array}{l}- \\
0.020\end{array}$ & $\begin{array}{l}0.00 \\
1\end{array}$ \\
\hline & & & & & & & & & 0.062 & \\
\hline $\begin{array}{l}\text { Secondary/ } \\
\text { higher }\end{array}$ & 31.52 & 49.42 & 21.7 & 39.21 & 41.17 & 20.7 & 3.1 & 0.000 & $\overline{-}-000$ & $\begin{array}{l}0.00 \\
0\end{array}$ \\
\hline & & & & & & & & & 0.006 & \\
\hline \multicolumn{11}{|c|}{ Household Head } \\
\hline Male & 40.03 & 40.21 & 21.4 & 19.29 & 39.25 & 21.4 & 3.6 & 0.004 & $\begin{array}{l}- \\
0.006\end{array}$ & $\begin{array}{l}0.61 \\
0 \\
\end{array}$ \\
\hline & & & & & & & & & 0.038 & \\
\hline Female & 31.81 & 60.71 & 19.7 & 21.6 & 31.61 & 21.5 & 13.2 & 0.007 & $\overline{-}-\overline{0}$ & $\begin{array}{l}0.15 \\
5\end{array}$ \\
\hline & & & & & & & & & 0.064 & \\
\hline \multicolumn{11}{|c|}{ HF nearest Distance } \\
\hline Huge Issue & 23.56 & 47.83 & 21.2 & 15.79 & 33.86 & 12.7 & 9 & 0.016 & $\overline{0}-017$ & 0.29 \\
\hline
\end{tabular}




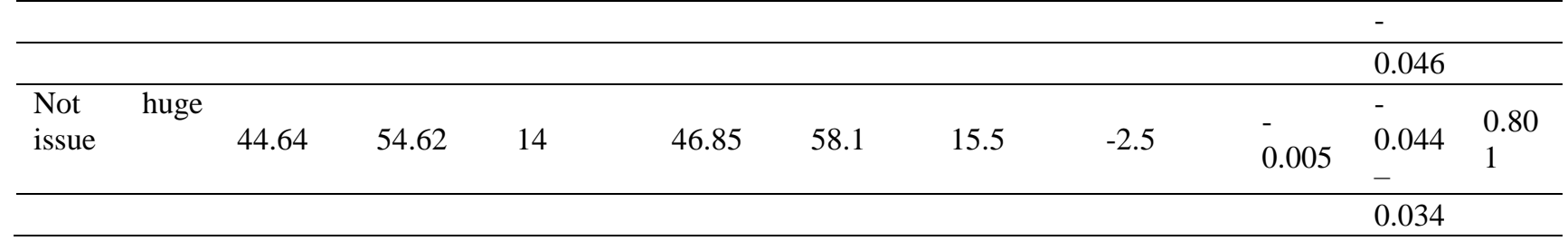

Regression of Binary and Multivariate analysis for minimum four ANC:

Table 4 represents the regression of binary and multivariate for at least four ANCs. In at least four ANC visits, the independent variables that contributed to the changes included wealth indicator's p-value 0.006, a p-value of ethnicities is 0.009), the p-value of gender is 0.001 and p-value of husband's education is 0.000 , and the p-value of the ecological region is 0.05. Minimum 4 ANC visits between 2017 and 2019 did not have an essential role in involvement, and the pvalue is 0.053 .

Table 4: Regression of Binary and Multivariate Analysis for minimum four ANC

\begin{tabular}{|c|c|c|c|c|c|c|c|c|}
\hline \multirow{4}{*}{ variables } & \multicolumn{8}{|c|}{ Data from 2016 to 2020} \\
\hline & \multicolumn{4}{|c|}{ Bivariate Analysis of coefficients } & \multicolumn{4}{|c|}{ Multivariate Analysis of coefficients } \\
\hline & \multirow[t]{2}{*}{ beta } & \multicolumn{2}{|l|}{ CI $(95 \%)$} & \multirow[t]{2}{*}{ p-val } & \multirow[t]{2}{*}{ beta } & \multicolumn{2}{|c|}{ CI $(95 \%)$} & \multirow[t]{2}{*}{ p-val } \\
\hline & & Low & Up & & & Low & $\mathrm{Up}$ & \\
\hline Wealth index & 0.1014 & 0.0644 & 0.1384 & 0.000 & 0.074 & 0.037 & 0.111 & 0.000 \\
\hline $\begin{array}{l}\text { Female } \\
\text { education }\end{array}$ & 0.1603 & 0.2130 & 0.2187 & 0.015 & 0.014 & 0.040 & 0.218 & 0.002 \\
\hline $\begin{array}{l}\text { Female } \\
\text { Occupation }\end{array}$ & 0.0341 & 0.0076 & 0.0607 & 0.012 & 0.025 & 0.002 & 0.049 & 0.036 \\
\hline $\begin{array}{l}\text { Place of } \\
\text { residence }\end{array}$ & -0.1436 & -0.2143 & -0.0728 & 0.000 & -0.085 & -0.145 & -0.024 & 0.006 \\
\hline Ethnicity & -0.2161 & -0.13780 & -0.30041 & 0.002 & -0.158 & -0.041 & -0.005 & 0.004 \\
\hline Household Gender & 0.06632 & 0.0093861 & 0.1056586 & 0.019 & 0.057 & 0.014 & 0.100 & 0.010 \\
\hline Organic area & -0.0413 & -0.083944 & -0.006248 & 0.033 & -0.022 & -0.089 & 0.005 & 0.051 \\
\hline Education of Husband & 0.025961 & 0.1326723 & 0.1055814 & 0.005 & 0.045 & 0.061 & 0.078 & 0.004 \\
\hline Year & & & & & 0.044 & 0.019 & 0.049 & 0.000 \\
\hline Involvement & & & & & 34.563 & -1.227 & 98.655 & 0.053 \\
\hline $\begin{array}{l}\text { Involvement• } \\
\text { Year }\end{array}$ & & & & & -0.034 & -0.056 & 0.004 & 0.053 \\
\hline Constant & & & & & -59.296 & 98.952 & 33.272 & 0.002 \\
\hline
\end{tabular}

Expert Delivery of Assisting Birth Analysis:

Table 5 represents the multivariate and binary regression analysis for the delivery of expert delivery of assisting Analysis. The independent variables contributing to SBA delivery changes included wealth, education for women, residence, the age for women, Distance from health facilities, and the green region. For expert delivery of assisting birth delivery between 2017 and 2019, involvement played no significant role.

Table 5: Regression Analysis of SBA

\begin{tabular}{|c|c|c|c|c|c|c|c|c|}
\hline \multirow{4}{*}{ Variables } & \multicolumn{8}{|c|}{ Period 2011 to 2016} \\
\hline & \multicolumn{4}{|c|}{ Bivariate Regression analysis } & \multicolumn{4}{|c|}{ Multivariate Regression Analysis } \\
\hline & \multirow[t]{2}{*}{ beta } & \multicolumn{2}{|l|}{ CI (95\%) } & \multirow[t]{2}{*}{ p-val } & \multirow[t]{2}{*}{ beta } & \multicolumn{2}{|c|}{ CI (95\%) } & \multirow[t]{2}{*}{ p-val } \\
\hline & & Lower & Upper & & & Lower & Upper & \\
\hline $\begin{array}{l}\text { Wealth } \\
\text { index }\end{array}$ & $\begin{array}{l}0.167824 \\
3\end{array}$ & $\begin{array}{l}0.131630 \\
7\end{array}$ & $\begin{array}{l}0.20401 \\
8\end{array}$ & 0.000 & 0.1246 & 0.0846 & 0.1646 & 0.000 \\
\hline $\begin{array}{l}\text { Women } \\
\text { education }\end{array}$ & $\begin{array}{l}0.162665 \\
8\end{array}$ & $\begin{array}{l}0.134411 \\
5\end{array}$ & 0.19092 & 0.000 & 0.0962 & 0.0650 & 0.1275 & 0.000 \\
\hline
\end{tabular}




\begin{tabular}{|c|c|c|c|c|c|c|c|c|}
\hline Place of residence & $\begin{array}{l}- \\
0.194510 \\
8\end{array}$ & -0.257598 & -0.13142 & 0.000 & -0.1268 & -0.1810 & -0.0726 & 0.000 \\
\hline Women age & $\begin{array}{l}0.109202 \\
8\end{array}$ & -0.141533 & -0.07687 & 0.000 & -0.0420 & -0.0742 & -0.0097 & 0.0110 \\
\hline Distance from $\mathrm{HF}$ & $\begin{array}{l}0.181869 \\
4\end{array}$ & 0.123608 & $\begin{array}{l}0.24013 \\
1\end{array}$ & 0.000 & 0.0757 & 0.0207 & 0.1308 & 0.0070 \\
\hline Ecological region & $\begin{array}{l}0.022300 \\
3\end{array}$ & -0.01663 & $\begin{array}{l}0.06123 \\
1\end{array}$ & 0.260 & -0.0384 & -0.0717 & -0.0051 & 0.024 \\
\hline Year & & & & & 0.0302 & 0.0169 & 0.0435 & 0.000 \\
\hline Involvement & & & & & -1.6231 & 45.8676 & 42.9974 & 0.854 \\
\hline $\begin{array}{l}\text { Intervention } \\
\text { - Year }\end{array}$ & & & & & 0006 & -0.0306 & .0309 & 0.833 \\
\hline Constant & & & & & 61.5864 & 81.3354 & 31.7754 & 0.001 \\
\hline
\end{tabular}

\section{DISCUSSION}

This Analysis aims to determine the involvement efficiency of the 16 districts compared to other districts. The study's main objective was to assess the response bundle's effect in Punjab using the Investment Case (IC) procedure (Prinja et al., 2015). In sub-Saharan African states, maternal and child healthcare factors are higher, with an increased proportion (Corsi \& Subramanian, 2014). There have been some changes in the at least 4 ANCs and qualified care providers, but these improvements are essentially identical in both involvement and contrast. The discrepancies in the Involvement and Contrast regions showed haven't noticeable change. This action played no essential action in any of the leading investment case metrics between 2017 and 2019. Studies in 54 countries found that more minor equal involvements were accompanied by four or more prenatal visits to eligible childbirth involvements (Ameyaw \& Dickson, 2020). A systematic meta-analysis of 11 randomized and clustered randomizations from countries such as Nepal, Bangladesh, India, and Pakistan shows that there has not any difference among the combined set involvement and control and three or more ANC visits and children's education (Colbourn et al., 2013). According to Baqui et al. (2016), In Bangladesh, a randomly controlled cluster study was carried out the bundle of public-based neonatal motherly involvement in the functional and awareness measures relating to improvement in maternal and neonate involvement in comparative areas was performed.

In contrast with the individual involvement programs, a systematic review of mother's health programs in resourcelimited countries found a substantial effect on maternal health. A research group in India has demonstrated essential changes in health and health care (Metwally et al., 2020). Also, in 259 tests and reports, which include systemic and descriptive Analysis, randomized controlled trials, classified, controlled, and regulated pre-post and uncontrolled timeseries studies, cross-sectional tests, and expert opinion documents, a systematic analysis that analyses 208 novel methods to MNH has completed creative technologies with inventive implants. Applied by the Government of Punjab to identify hurdles in recuperating maternal health services in Punjab (MHS), the Punjab Safe Motherhood Initiative (PSMI) Project is a successful well-coordinated program to reduce the blocks within the health and financial state services.

\section{INVOLVEMENT}

The involvement structure already constitutes a complex project involving multiple approaches to various motherly, newborn, and child healthcare measures. The work comprises all the factors that make it difficult and complicated to target healthcare professionals in the Tanahashi model. Findings from various tests and studies were done in all the other countries may lead to investment cases, and the situations may not have the same as in neighboring states. The investment case involves all health stakeholders in the districts. Suppose there are many treatments in the equipment. In this situation, the entire involvement has been diminished because of service providers' dispersed focus and policymakers, and judgment creators. An appraisal of the Asia-Pacific IC has also shown how complicated the District Managers' approach is over and above the staff capability.

\section{IMPLEMENTATION OF INTENSITY AND QUALITY}

There was a greater emphasis on budgeting and technical support in the districts when carrying out an investment event. The district workshop concluded with action plans with different stakeholder roles for various tracers. However, both stakeholders and implementing partners have not monitored the action plans regularly. In the districts, there were no technicians who monitored the involvement regularly. The restriction of resources was also an essential factor in the participants' quality since minimal resources (mainly staff) were available for the hard-to-access activities.

\section{CONCLUSION}

The structure, workforce, and time frame of the study are significant factors in the framework to show the possible 
impact on communities. Thrift Data from services and health surveys were obtained. The study used assessed approximately experimental results using distinctions and multivariable linear regression. To evaluate the effects of an investment in the Punjab districts, the study used PDHS data of 2017 and 2019. A significant increase has been shown from the 2017 and 2019 Punjab Health and Demography Survey. Certain variations were observed in essential facts such as a minimum of 4 ANCs and qualified health workers, but these enhancements are generally identical in both participation and contrast regions. The results have shown that participation methods need to be redesigned so that the IC approach is efficient.

\section{LIMITATION AND STUDY FORWARD}

Since the study is almost experimental, various potential biases may be present. The MICS samples cannot be taken to account for the entire district population since some district samples are more extensive and some may have sample data. The people from each study area may vary. The data for the respective MNCH indicators is smaller, which reduces the analysis frequency and impacts the strong relation of variables. The districts selected as population contrasts had almost the same HDI (Human Development Index) as the involvement regions; however, it is comparatively easy to achieve. By IC method, all districts not accessible were selected as districts of involvement. Therefore, the interference and contrast districts are only - this study first time analyzing the impact of investment case in the RMNCAH program in Punjab. The research will provide a proper guideline for the policymaker to design a needbased policy for good entree to motherly and child healthcare facilities.

\section{ACKNOWLEDGEMENT}

Thank you to all my colleagues who have been so supportive on the way to research. I would like to take this opportunity to thank you warmly. I would also like to thank my family most sincerely for their support during the entire lifetime and primarily through the research paper. I have the opportunity to finish this paper because of their unconditional love and prayers. Last but not least, thanks to all those who participated in this research.

\section{AUTHORS CONTRIBUTION}

Majid Ali and Naveed conceived the presented concept. Majid Ali was the one who devised the idea and carried out the calculations. Ali verified the analytical methods. All authors contributed to the final manuscript by discussing the findings. Adnan gave valuable comments on the paper.

\section{REFERENCES}

1. Ahinkorah, B. O., Seidu, A. A., Agbaglo, E., Adu, C., Budu, E., Hagan, J. E., ... \& Yaya, S. (2021). Determinants of antenatal care and skilled birth attendance services utilization among childbearing women in Guinea: evidence from the 2018 Guinea Demographic and Health Survey data. BMC Pregnancy and Childbirth, 21(1), 1-11. https://doi.org/10.1186/s12884-020-03489-4

2. Ameyaw, E. K., \& Dickson, K. S. (2020). Skilled birth attendance in Sierra Leone, Niger, and Mali: Analysis of demographic and health surveys. BMC public health, 20(1), 164. https://doi.org/10.1186/s12889-020-8258-Z

3. Askew, D. A., Guy, J., Lyall, V., Egert, S., Rogers, L., Pokino, L. A., ... \& Schluter, P. J. (2019). A mixedmethods exploratory study is tackling smoking during pregnancy in an urban Aboriginal and Torres Strait Islander primary health care service. BMC public health, 19(1), 1-10. https://doi.org/10.1186/s12889-0196660-1

4. Azad, G. F., Minton, K. E., Mandell, D. S., \& Landa, R. J. (2021). Partners in School: An Implementation Strategy to Promote Alignment of Evidence-Based Practices Across Home and School for Children with Autism Spectrum Disorder. Administration and Policy in Mental Health and Mental Health Services Research, 48(2), 266-278. https://doi.org/10.1007/s10488-020-01064-9

5. Baqui, A. H., Williams, E., El-Arifeen, S., Applegate, J. A., Mannan, I., Begum, N., ... \& Darmstadt, G. L. (2016). Effect of community-based newborn care on cause-specific neonatal mortality in Sylhet district, Bangladesh: findings of a cluster-randomized controlled trial. Journal of Perinatology, 36(1), 71-76. https://doi.org/10.1038/jp.2015.139

6. Bärnighausen, T., Tugwell, P., Røttingen, J. A., Shemilt, I., Rockers, P., Geldsetzer, P., ... \& Atun, R. (2017). Quasi-experimental study designs series - paper 4: uses and value. Journal of clinical epidemiology, 89, 21-29. https://doi.org/10.1016/j.jclinepi.2017.03.012

7. Bhutta, Z. A., Hafeez, A., Rizvi, A., Ali, N., Khan, A., Ahmad, F., ... \& Jafarey, S. N. (2013). Reproductive, maternal, newborn, and child health in Pakistan: challenges and opportunities. The Lancet, 381(9884), 22072218. https://doi.org/10.1016/S0140-6736(12)61999-0

8. Cabero-Roura, L., \& Rushwan, H. (2014). An update on maternal mortality in low-resource countries. International Journal of Gynecology \& Obstetrics, 125(2), 175-180. https://doi.org/10.1016/j.ijgo .2014 .02 .002

9. Colbourn, T., Nambiar, B., Bondo, A., Makwenda, C., Tsetekani, E., Makonda-Ridley, A., ... \& Costello, A. (2013). Effects of quality improvement in health facilities and community mobilization through women's groups on maternal, neonatal, and perinatal mortality in three Malawi districts: MaiKhanda, a cluster 
randomized controlled effectiveness trial. International health, 5(3), 180-195. https://doi.org/10.1093/inth ealth/iht011

10. Corsi, D. J., \& Subramanian, S. V. (2014). Association between coverage of maternal and child health involvements, and under-5 mortality: a repeated cross-sectional analysis of 35 sub-Saharan African countries. Global health action, 7(1), 24765. https://doi.org/10.3402/gha.v7.24765

11. Dawson, A. J., Buchan, J., Duffield, C., Homer, C. S., \& Wijewardena, K. (2014). Task shifting and sharing in maternal and reproductive health in low-income countries: a narrative synthesis of current evidence. Health policy and planning, 29(3), 396-408. https://doi.org/10.1093/heapol/czt026

12. El-Kak, F., Kabakian-Khasholian, T., Ammar, W., \& Nassar, A. (2020). A review of maternal mortality trends in Lebanon, 2010-2018. International Journal of Gynecology \& Obstetrics, 148(1), 14-20. https://doi.org/1 0.1002/ijgo.12994

13. Fotso, J. C., Higgins-Steele, A., \& Mohanty, S. (2015). Male engagement as a strategy to improve utilization and community-based delivery of maternal, newborn and child health services: evidence from an involvement in Odisha, India. BMC health services research, 15(1), 1-10. https://doi.org/10.1186/1472-6963-15-S1-S1

14. Garcia, A., Orengo, H. A., Conesa, F. C., Green, A. S., \& Petrie, C. A. (2019). Remote sensing and historical morphodynamics of alluvial plains. The 1909 indus flood and the city of Dera Ghazi Khan (province of Punjab, Pakistan). Geosciences, 9(1), 21. https://doi.org/10.3390/geosciences 9010021

15. Gardezi, N. U. Z. (2021). Public health insurance and birth outcomes: evidence from Punjab, Pakistan. Health Policy and Planning, 36(1), 1-13. https://doi.org/10.1093/heapol/czaa115

16. Geller, S. E., Koch, A. R., Garland, C. E., MacDonald, E. J., Storey, F., \& Lawton, B. (2018). A global view of severe maternal morbidity: moving beyond maternal mortality. Reproductive health, 15(1), 31-43. https://doi.org/10.1186/s12978-018-0527-2

17. Hao, W., Shah, S. M. A., Nawazb, A., Barkat, M. Q., \& Souhail, A. (2020). COVID-19 Epidemic Spread and the Impact on Public Health \& Safety Policy: An Analysis of the Adoption of Preventive Measures and Effective Management: Evidence from Pakistan. Revista Argentina de Clínica Psicológica, 29(4), 722-736.

18. Hashmi, A., Amirah, N., \& Yusof, Y. (2021). Organizational performance with disruptive factors and inventory control as a mediator in public healthcare of Punjab, Pakistan. Management Science Letters, 11(1), 77-86. https://doi.org/10.5267/j.msl.2020.8.028

19. Heerdegen, A. C. S., Gerold, J., Amon, S., Agyemang, S. A., Aikins, M., \& Wyss, K. (2020). How Does District Health Management Emerge Within a Complex Health System? Insights for Capacity Strengthening in Ghana. Frontiers in Public Health, 8. https://doi.org/10.3389/fpubh.2020.00270

20. Jin, Y., Mankadi, P. M., Rigotti, J. I., \& Cha, S. (2018). Cause-specific child mortality performance and contributions to all-cause child mortality, and number of child lives saved during the Millennium Development Goals era: a country-level analysis. Global health action, 11(1), 1546095. https://doi.org/10.1080/16549 716.2018.1546095

21. Khan, S., Haider, S. I., \& Bakhsh, R. (2020). Socio-Economic and Cultural Determinants of Maternal and Neonatal Mortality in Pakistan. Global Regional Review, 1, 1-7. https://doi.org/10.31703/grr.2020(V-I).01

22. Laing, S. K., Griffiths, U., Raza, A. A., Zulu, F., Yakubu, A., Bessias, S., \& Ozawa, S. (2020). An investment case for maternal and neonatal tetanus elimination. Vaccine,38(9), 2241-2249. https://doi.org/10.1016 j.vaccine.2019.11.052

23. Liang, J., Li, X., Kang, C., Wang, Y., Kulikoff, X. R., Coates, M. M., .. \& Wang, H. (2019). Maternal mortality ratios in 2852 Chinese counties, 1996-2015, and achievement of Millennium Development Goal 5 in China: a subnational analysis of the Global Burden of Disease Study 2016. The Lancet, 393(10168), 241-252. https://doi.org/10.1016/S0140-6736(18)31712-4

24. Magni, G. (2017). Indigenous knowledge and implications for the sustainable development agenda. European Journal of Education, 52(4), 437-447. https://doi.org/10.1111/ejed.12238

25. Mauricio Marrufo, R. (2018). Changes in government and its impact on urban, economic, and health policies in the Paso del Norte Region. Producto de investigación ICB.

26. McCollum, R., Taegtmeyer, M., Otiso, L., Mireku, M., Muturi, N., Martineau, T., \& Theobald, S. (2019). Healthcare equity analysis: applying the Tanahashi model of health service coverage to community health systems following devolution in Kenya. International journal for equity in health, 18(1), 1-12. https://doi.org/10.1186/s12939-019-0967-5

27. Metwally, A. M., Abdel-Latif, G. A., Mohsen, A., El Etreby, L., Elmosalami, D. M., Saleh, R. M., ... \& Abdelrahman, M. (2020). Strengths of community and health facilities based involvements in improving women and adolescents' care seeking behaviors as approaches for reducing maternal mortality and improving birth outcome among low income communities of Egypt. BMC Health Services Research, 20(1), 1-14. https://doi.org/10.1186/s12913-020-05412-1

28. Mumtaz, Z., Salway, S., Bhatti, A., Shanner, L., Zaman, S., Laing, L., \& Ellison, G. T. (2014). Improving maternal health in Pakistan: toward a deeper understanding of the social determinants of poor women's access to maternal health services. American journal of public health, 104(S1), S17-S24. https://doi.org/10.2105/AJP H.2013.301377 
29. Mustafa, M., Batool, A., Fatima, B., Nawaz, F., Toyama, K., \& Raza, A. A. (2020, April). Patriarchy, maternal health and spiritual healing: Designing maternal health involvements in Pakistan. In Proceedings of the 2020 CHI Conference on Human Factors in Computing Systems (pp. 1-13). https://doi.org/10.1145/3 313831.3376294

30. Nigussie, K., Degu, G., Chanie, H., \& Edemealem, H. (2021). Magnitude of Unintended Pregnancy and Associated Factors Among Pregnant Women in Debre Markos Town, East Gojjam Zone, Northwest Ethiopia: A Cross-Sectional Study. International Journal of Women's Health, 13, 129. https://doi.org/10.2147/ IJWH.S275346

31. Nisar, Y. B., \& Dibley, M. J. (2014). Determinants of neonatal mortality in Pakistan: secondary Analysis of Pakistan Demographic and Health Survey 2006-07. BMC Public Health, 14(1), 1-12. https://doi.org/10.1186 11471-2458-14-663

32. Novignon, J., Ofori, B., Tabiri, K. G., \& Pulok, M. H. (2019). Socioeconomic inequalities in maternal health care utilization in Ghana. International journal for equity in health, 18(1), 1-11. https://doi.org/10.11 86/s12939-019-1043-X

33. Okoli, C., Hajizadeh, M., Rahman, M. M., \& Khanam, R. (2020). Geographical and socioeconomic inequalities in the utilization of maternal healthcare services in Nigeria: 2003-2017. BMC Health Services Research, 20(1), 1-14. https://doi.org/10.1186/s12913-020-05700-w

34. Panda, B. K., Kumar, G., \& Awasthi, A. (2020). District level inequality in reproductive, maternal, neonatal and child health coverage in India. BMC public health, 20(1), 1-10. https://doi.org/10.1186/s12889-020-8151-9

35. Patel, B. B., Gurmeet, P., Sinalkar, D. R., Pandya, K. H., Mahen, A., \& Singh, N. (2016). A study on knowledge and practices of antenatal care among pregnant women attending antenatal clinic at a Tertiary Care Hospital of Pune, Maharashtra. Medical Journal of Dr. DY Patil University, 9(3), 354. https://doi.org/ $\underline{10.4103 / 0975-2870.182507}$

36. Perry, H. B., Rassekh, B. M., Gupta, S., \& Freeman, P. A. (2017). Comprehensive review of the evidence regarding the effectiveness of community-based primary health care in improving maternal, neonatal and child health: 7. shared characteristics of projects with evidence of long-term mortality impact. Journal of global health, 7(1). https://doi.org/10.7189/jogh.07.010907

37. Prinja, S., Bahuguna, P., Gupta, R., Sharma, A., Rana, S. K., \& Kumar, R. (2015). Coverage and financial risk protection for institutional delivery: how universal is provision of maternal health care in India?. PloS one, 10(9), e0137315. https://doi.org/10.1371/journal.pone.0137315

38. Requejo, J., Diaz, T., Park, L., Chou, D., Choudhury, A., Guthold, R., ... \& Banerjee, A. (2020). Assessing coverage of involvements for reproductive, maternal, newborn, child, and adolescent health and nutrition. bmj, 368. https://doi.org/10.1136/bmj.16915

39. Smith, E., Fazeli, F., Wilkinson, K., \& Clark, C. C. (2021). Physical behaviors and fundamental movement skills in British and Iranian children: An isotemporal substitution analysis. Scandinavian Journal of Medicine \& Science in Sports, 31(2), 398-404. https://doi.org/10.1111/sms.13837

40. Thapa, J. K., Stöckl, D., Sangroula, R. K., Thakur, D. N., Mehata, S., Pun, A., \& Delius, M. (2020). Impact of Investment Case on Equitable Access on Maternal and Child Health Services in Nepal: Quasi-experimental study. https://doi.org/10.21203/rs.3.rs-124408/v1

41. Thompson, C. G., Kim, R. S., Aloe, A. M., \& Becker, B. J. (2017). Extracting the variance inflation factor and other multicollinearity diagnostics from typical regression results. Basic and Applied Social Psychology, 39(2), 81-90. https://doi.org/10.1080/01973533.2016.1277529

42. Thompson-Dudiak, M. (2021). The Black Maternal Health Crisis: How to Right a Harrowing History through Judicial and Legislative Reform. DePaul J. Soc. Just., 14, 1.

43. Tibeihaho, H., Nkolo, C., Onzima, R. A., Ayebare, F., \& Henriksson, D. K. (2021). Continuous quality improvement as a tool to implement evidence-informed problem solving: experiences from the district and health facility level in Uganda. BMC Health Services Research, 21(1), 1-11. https://doi.org/10.1186/s12913021-06061-8

44. Tsolaki, A. C., Tsolaki, M., Pandria, N., Lazarou, E., Gkatzima, O., Zilidou, V., ... \& Bamidis, P. D. (2020). Web-based involvement effects on mild cognitive impairment based on apolipoprotein e genotype: Quasiexperimental study. Journal of medical Internet research, 22(5), e14617. https://doi.org/10.2196/14617

45. Viswanath, K., \& Kreuter, M. W. (2007). Health disparities, communication inequalities, and eHealth. American journal of preventive medicine,32(5), S131-S133. https://doi.org/10.1016 j.amepre.2007.02.012

46. Vogel, J. P., Bohren, M. A., Tunçalp, Ö., Oladapo, O. T., \& Gülmezoglu, A. M. (2016). Promoting respect and preventing mistreatment during childbirth. BJOG: An International Journal of Obstetrics \& Gynaecology, 123(5), 671-674. https://doi.org/10.1111/1471-0528.13750 